\title{
ARETT: Augmented Reality Eye Tracking Toolkit for Head Mounted Displays
}

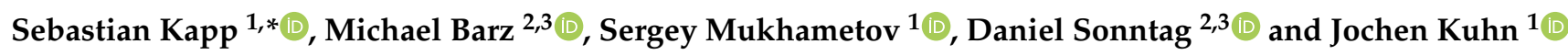 \\ 1 Department of Physics, Technische Universität Kaiserslautern, Erwin-Schrödinger-Str. 46, \\ 67663 Kaiserslautern, Germany; mukhamet@physik.uni-kl.de (S.M.); kuhn@physik.uni-kl.de (J.K.) \\ 2 German Research Center for Artificial Intelligence (DFKI), Interactive Machine Learning Department, \\ Stuhlsatzenhausweg 3, Saarland Informatics Campus D3_2, 66123 Saarbrücken, Germany; \\ michael.barz@dfki.de (M.B.); daniel.sonntag@dfki.de (D.S.) \\ 3 Applied Artificial Intelligence, Oldenburg University, Marie-Curie Str. 1, 26129 Oldenburg, Germany \\ * Correspondence: kapp@physik.uni-kl.de
}

Citation: Kapp, S.; Barz, M.; Mukhametov, S.; Sonntag, D.; Kuhn, J. ARETT: Augmented Reality Eye Tracking Toolkit for Head Mounted Displays. Sensors 2021, 21, 2234. https://doi.org/10.3390/s21062234

Academic Editor: Jamie A Ward

Received: 25 February 2021

Accepted: 17 March 2021

Published: 23 March 2021

Publisher's Note: MDPI stays neutral with regard to jurisdictional claims in published maps and institutional affiliations.

Copyright: (c) 2021 by the authors. Licensee MDPI, Basel, Switzerland. This article is an open access article distributed under the terms and conditions of the Creative Commons Attribution (CC BY) license (https:// creativecommons.org/licenses/by/ $4.0 /)$.

\begin{abstract}
Currently an increasing number of head mounted displays (HMD) for virtual and augmented reality (VR/AR) are equipped with integrated eye trackers. Use cases of these integrated eye trackers include rendering optimization and gaze-based user interaction. In addition, visual attention in VR and AR is interesting for applied research based on eye tracking in cognitive or educational sciences for example. While some research toolkits for VR already exist, only a few target AR scenarios. In this work, we present an open-source eye tracking toolkit for reliable gaze data acquisition in AR based on Unity 3D and the Microsoft HoloLens 2, as well as an R package for seamless data analysis. Furthermore, we evaluate the spatial accuracy and precision of the integrated eye tracker for fixation targets with different distances and angles to the user $(n=21)$. On average, we found that gaze estimates are reported with an angular accuracy of 0.83 degrees and a precision of 0.27 degrees while the user is resting, which is on par with state-of-the-art mobile eye trackers.
\end{abstract}

Keywords: augmented reality; eye tracking; toolkit; accuracy; precision

\section{Introduction}

Head mounted displays (HMD) got more affordable and lightweight in the last few years facilitating a broader usage of virtual and augmented reality (VR/AR) applications. In addition, recent devices are equipped with integrated eye trackers which primarily target novel gaze-based interaction techniques [1,2] and optimizing the display quality, e.g., using foveated rendering [3,4]. This creates new opportunities for eye tracking research in mixed reality settings. However, the number and functionality of research tools for AR and VR eye tracking is still limited, e.g., compared to the well-established stationary eye trackers that are attached to a two-dimensional display. Available commercial solutions for HMD eye tracking are mostly limited to VR (see, e.g., References [5,6]). Pupil Labs [6] offers an extension for AR eye tracking which consists of mobile eye tracking equipment attached to an HMD, but with only a loose integration into AR application development tools.

In this work, we aim at closing the gap of research tools for AR eye tracking. We implement an open-source toolkit that facilitates eye tracking research in AR environments with the Microsoft HoloLens 2. Our toolkit includes a package for the Unity 3D game development engine which enables simple integration of reliable gaze and meta data recordings in AR applications, and an $\mathrm{R}$ package for seamless post-hoc processing and analysis of the data. In addition, we conduct a user study $(n=21)$ for evaluating the spatial accuracy and precision of the gaze signal retrieved from our toolkit. We discuss our results and compare them to results for state-of-the-art mobile eye trackers from the literature. 


\section{Related Work}

Our work is related to other research-oriented toolkits and software solutions for headmounted eye tracking systems, particularly to those targeting VR and AR environments, and to literature on measuring the gaze estimation error.

\subsection{AR and VR Eye Tracking}

Some toolkits for eye tracking research in VR are available. Tobii offers a solution for eye tracking analysis in VR by providing tools for the integration of eye tracking hardware to HMDs and analysis software for eye tracking research [7]. Another commercial eye tracking add-on is offered by Pupil Labs for the HTC Vive HMD together with opensource software for data analysis [6]. Non-commercial frameworks for eye tracking in AR or VR exist, as well. Stratmann et al. [8] presented EyeMR, a low-cost system for integrating eye tracking into VR based on the Pupil Capture software and a custom Unity 3D framework. Lee et al. [9] also presented a method for low-cost gaze tracking and gaze point estimation in head-mounted devices. Mardanbegi and Pfeiffer [10] presented the EyeMRTK toolkit to develop gaze-based interaction techniques in VR and AR; however, the current implementation is limited to specific VR headsets. Adhanom et al. [11] presented the GazeMetrics tool which provides a standardized approach to measure accuracy and precision in VR settings.

The range of AR eye tracking toolkits is more limited. Pupil Labs [6] offers eye tracking add-ons for the Microsoft HoloLens 1 and the Epson Moverio BT-300, but the analysis software is tailored to mobile eye tracking without HMDs and their integration into the Unity 3D development environment is discontinued (https://github.com/pupil-labs/ hmd-eyes/issues/100\#issuecomment-662362737, accessed on 20 November 2020). This limits the usefulness of the offered add-ons and restricts applications to use cases in which no AR integration is required. Recent HMDs, like the Magic Leap 1 [12] and the Microsoft HoloLens 2 [13], are equipped with integrated eye trackers. However, the toolkits and APIs provided by the manufacturers are targeted at gaze-based interaction and not at eye tracking research $[14,15]$. Still, this enables an easy integration of visual attention into AR applications: using the spatial awareness of the devices provides eye-in-world data which otherwise has to be integrated using additional sensors [16]. We build our toolkit on top of the eye tracking APIs of the HoloLens 2 device [13]. However, all device specific code is encapsulated in a data access layer which enables easy adaption of the toolkit to other eye tracking enabled AR devices.

\subsection{Measuring the Gaze Estimation Error}

Eye tracking research studies investigate the impact of an intervention on the eye movements of a participant. Typically, the gaze samples or fixations, i.e., the periods for which the eye is relatively still, are used to approximate the human visual attention, and are mapped to areas of interest (AOIs) for analysis. High gaze estimation quality is essential for eye tracking research because errors can heavily undermine the results [17]. However, a key problem in head-mounted eye tracking is that the gaze estimation error, i.e., the difference between the estimated and true gaze position, can be substantial, particularly if participants move and if fixation distances vary $[18,19]$. Besides user position and orientation, also factors specific to the eye tracker and display, e.g., parameters of the calibration routine and of the display detection algorithm, can have significant impact on the gaze estimation error [20]. Typical metrics for the error of gaze estimation include spatial accuracy and spatial precision [21]. Spatial accuracy is commonly computed as the mean angular deviation of fixations to the actual position, and spatial precision as the root mean square error or standard deviation of individual gaze samples from their centroid [21,22]. 


\section{Augmented Reality Eye Tracking Toolkit}

We develop an eye tracking toolkit for augmented reality applications using the Unity $3 \mathrm{D}$ game development engine [23]. Our goal is to simplify the access to eye tracking data from the Microsoft HoloLens 2 for research purposes or advanced interaction techniques. We aim at providing raw gaze data robustly at a fixed data rate, without delay, and with highest possible spatial accuracy and precision. For this, we implement an easyto-use interface to control recordings and enable a simple integration into applications and research studies. In addition, we implement a package for the statistical computing environment $\mathrm{R}$ for seamless data analysis [24]. The toolkit, a detailed documentation, and an example project are available on GitHub (https:/ / github.com/AR-Eye-TrackingToolkit/ARETT, accessed on 22 March 2021) under the MIT open-source license.

\subsection{Overview of HoloLens 2 Technology}

We briefly summarize the underlying technology, i.e., the eye tracking hardware and software of the HoloLens 2 which we interface in our data access layer. Similar to other head-mounted eye trackers, the Microsoft HoloLens 2 uses two infrared cameras that yield a close-up view of the wearer's eyes [13]. After using the built-in 9-point calibration routine, a closed processing module provides real-time 3D gaze data to developers including a gaze origin and a direction vector. Gaze data can be accessed within Unity 3D via the Mixed Reality Toolkit (MRTK) [14] and via the underlying API for the Universal Windows Platform (UWP) [25]. The MRTK primarily focuses on enabling gaze-based interaction via an easy-to-use API for developers. It does not offer recordings for research purposes, nor does it guarantee a fixed sampling rate which is tied to the Unity3D update rate. Hence, gaze samples might be missed. Our system is based on the API for the UWP which provides unsmoothed data, more stable data rates, and a higher level of control. Further, a high precision timestamp in the system-relative QueryPerformanceCounter (QPC) time domain with a precision of $100 \mathrm{~ns}$ is provided for each data point. The manufacturer is vague in reporting specifications related to data quality: the data rate is "approximately $30 \mathrm{~Hz}^{\prime \prime}$ with a spatial accuracy that ranges "approximately within 1.5 degrees" [26].

\subsection{Architecture \& Components of the Recording Tool}

The recording tool of our toolkit is implemented as a package for the Unity 3D game development engine and includes four major components: the generic data provider with the HoloLens-specific data access layer that makes timestamped gaze data available in realtime, the data logger that is responsible for storing the data, the web-based control interface, and a set of utility tools for data visualization. An overview of our system's architecture and the interplay of individual components is shown in Figure 1. In the following, we describe each component in detail and discuss the implementation of egocentric video capture.

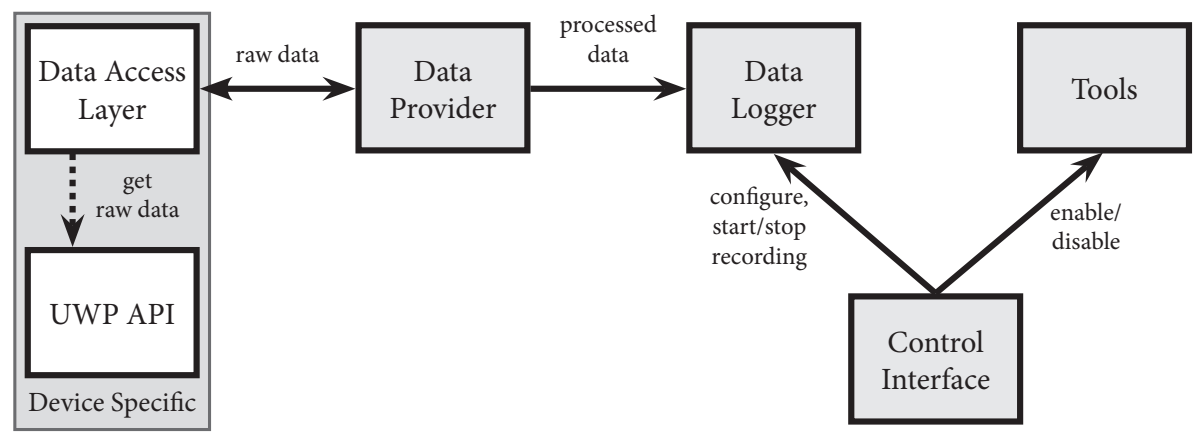

Figure 1. A diagram visualizing the components of the toolkit and their interaction. 
The data provider accesses raw eye tracking data using the data access layer, processes it and raises according gaze data events. The data access layer on the HoloLens 2 checks for new gaze samples in a separate thread every $10 \mathrm{~ms}$ to reliably obtain all gaze samples from the API with a supposed data rate of $30 \mathrm{~Hz}$, i.e., we expect a new gaze sample every $33.33 \mathrm{~ms}$. This pulling is necessary as no new data event is provided by the API. Each gaze sample includes the origin of the gaze point, its direction vector, and a timestamp. All gaze samples received by the access layer are queued in the data provider and processed in the next frame update in the Unity 3D main thread. For each gaze sample, we cast a ray and check for hits with collider objects in the scene. If the option spatial mapping of the MRTK is enabled for the application, this includes the real environment that is scanned by the depth sensors of the HoloLens 2 . If a collider is hit, we extend the gaze sample by the intersection coordinates in the world coordinate system, the object's name, position, rotation and scale, the intersection point in the object's local coordinate system, and the gaze point projection to the $2 \mathrm{D}$ eye displays. In addition, we support AOI colliders for real-time gaze-to-AOI mapping with support for dynamic AOIs. AOI collider objects can be placed at any position of a Unity 3D scene or attached to virtual objects in the scene. AOIs must be defined during the application development phase. Real-time and gaze-based adaptations can be realized using custom scripts. Synchronized recordings of the gaze signal and the front-facing camera can be used to define further AOIs post-hoc. We separately cast gaze rays to check for hits with AOI colliders. In addition, we offer an option to store the position, rotation and scaling of game objects in the scene in our gaze sample. This can be used to simulate or visualize sequences of interest post-hoc. For each processed sample, we raise an event that can be subscribed by other components, such as the data logger.

The data logger component provides the option to record all gaze samples. An overview of all recorded data columns can be found in Table 1 . The files are named based on the participant's pseudonym and a custom recording name. All recordings of a participant are stored in one folder. The gaze data samples are saved as comma separated values (CSV) with one sample per row and the columns as described in Table 1. In addition, we store meta information of the recording, e.g., the start and end time of the recording, in a separate text file in the JSON format. After the recording is started, developers can log additional events in terms of an info string that is stored as part of the gaze sample and in the JSON file. This enables researchers to track custom interaction events, which are of interest to their research question, and session annotations. The recording can be started via function calls and is used in our web-based control interface.

We integrate two utility tools that ease the development, debugging, and monitoring of study prototypes. This includes a tool for visualizing a grid of fixation targets, and one for highlighting AOIs. The grid of fixation targets enables easy collection of gaze samples and corresponding target positions for the evaluation of spatial accuracy and precision. We use this tool in our evaluations: we show nine fixation targets arranged in a $3 \times 3$ grid at multiple distances from the user. The AOI highlighting helps in debugging dynamic and interactive experiment scenes in which AOIs can move around, appear and disappear during the experiment session. For this, the developer can add custom visualizations which can be dynamically shown and hidden using the control interface.

Our toolkit comes with a web-based control interface (see Figure 2). It enables the experimenter to easily set a participant acronym and a recording name, and to start and stop recordings from any computer in the local network. Further, it provides access to our utility tools and allows an experimenter to add custom annotations to the recording during the study. 
Table 1. Overview of recorded data.

\begin{tabular}{|c|c|}
\hline Data Column & Description \\
\hline \multicolumn{2}{|l|}{ Time data } \\
\hline eyeDataTimestamp & Unix timestamp of the gaze data (in ms) \\
\hline eyeDataRelativeTimestamp & Relative timestamp of the gaze data (in ms, $100 \mathrm{~ns}$ precision) \\
\hline frameTimestamp & Unix timestamp of the frame in which the data was processed (in ms) \\
\hline \multicolumn{2}{|l|}{ Gaze data } \\
\hline isCalibrationValid & Flag if the calibration of the wearer is valid \\
\hline gazeHasValue & Flag if valid gaze data exists (origin/direction) \\
\hline gazeOrigin_(x/y/z) & Gaze origin in the global reference frame \\
\hline gazeDirection_(x/y/z) & Gaze direction in the global reference frame \\
\hline gazePointHit & Flag if the raycast hit an object and a gaze position exists \\
\hline gazePoint_(x/y/z) & Position of the gaze point in the global reference frame \\
\hline gazePoint_target_name & Name of the game object hit by the gaze ray \\
\hline gazePoint_target_(x/y/z) & Position of the gaze point in the local reference frame of the hit object \\
\hline gazePoint_target_(pos/rot/scale)_(x/y/z) & Position, rotation, and scale of the game object hit by the gaze ray \\
\hline gazePoint(Left/Right/Mono)Screen_( $x, y, z)$ & Position of the gaze point on the left, right and virtual mono display \\
\hline gazePointWebcam_(x,y,z) & Position of the gaze point on the webcam image \\
\hline \multicolumn{2}{|l|}{ AOI data } \\
\hline gazePointAOIHit & Flag if the gaze ray hit an AOI \\
\hline gazePointAOI_(x/y/z) & Position of the gaze point on the AOI in global coordinates \\
\hline gazePointAOI_target_name & Name of the game object representing the AOI \\
\hline gazePointAOI_target_(x/y/z) & Position of the gaze point in the local reference frame of the AOI \\
\hline gazePointAOI_target_(pos/rot/scale)_(x/y/z) & Position, rotation, and scale of the game object hit by the AOI ray \\
\hline gazePointAOIWebcam_(x,y,z) & Position of the gaze point on the AOI on the webcam image \\
\hline \multicolumn{2}{|l|}{ Additional information } \\
\hline gameObject_objectName_(pos/rot/scale)_(x/y/z) & Position, rotation, and scale of selected game objects \\
\hline info & Info string of a logged event \\
\hline
\end{tabular}
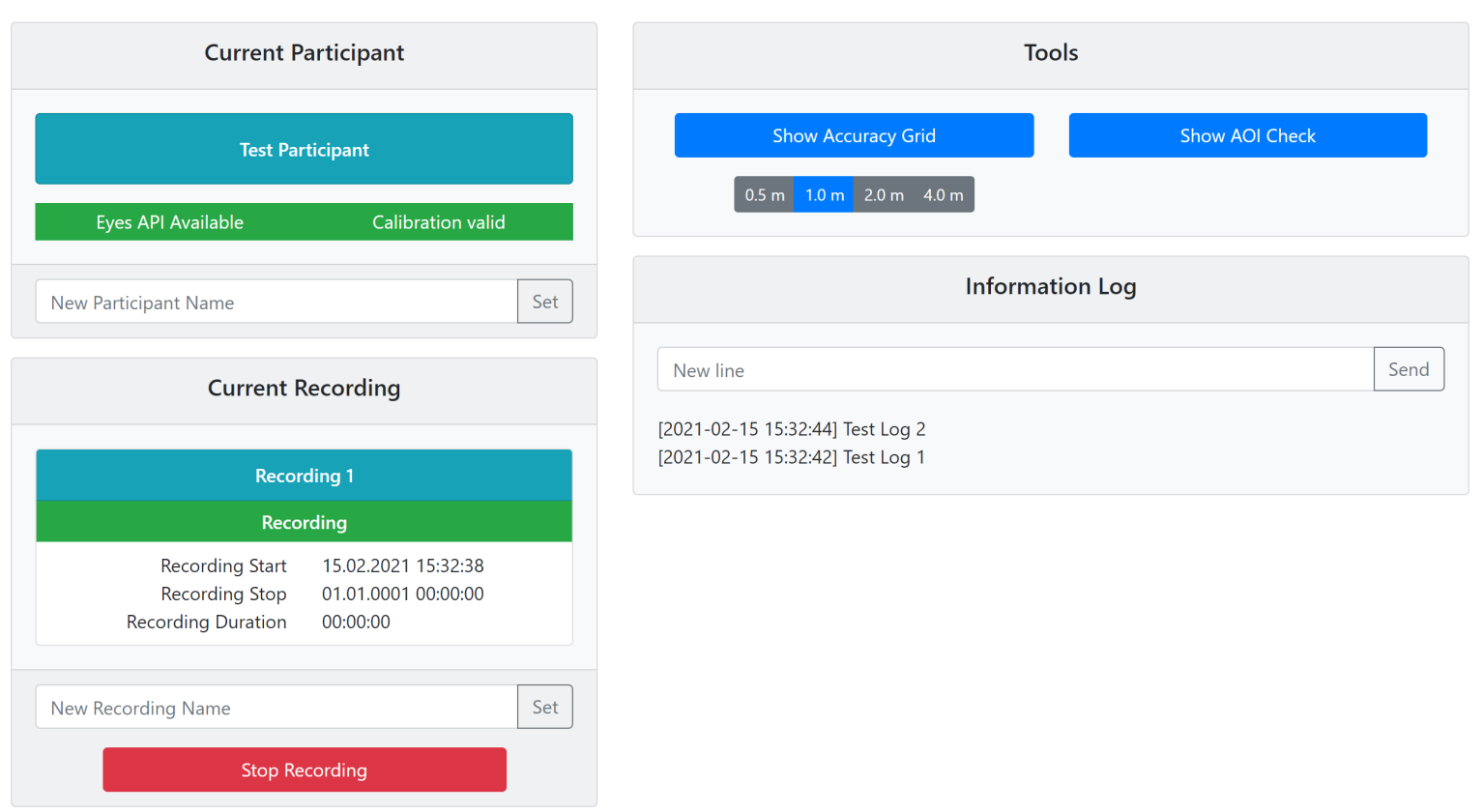

Figure 2. Screenshot of the control interface accessible over the network. 
Typically, head-mounted eye trackers use a world camera to record the environment from an egocentric perspective and map the wearer's pupil positions to the corresponding video frames. The integrated eye tracker of the Microsoft HoloLens 2, however, maps pupil positions to gaze rays in the $3 \mathrm{D}$ coordinate system of the device.

Our toolkit adds a virtual camera to the 3D scene that matches the location, projection, and resolution of the integrated front-facing camera. This enables the projection of the 3D gaze position to the virtual 2D camera image and, hence, to the webcam image. The virtual camera is preconfigured to match the integrated, front-facing webcam of the HoloLens 2. We recommend to check the configuration per use case and to adapt it, if the camera specifications differ. The 2D gaze signal is reported via the gaze sample event of the data provider and recorded in the gazePointWebcam column.

If video streaming or capturing for demonstration purposes is required only, the Mixed Reality Capture (MRC) module of HoloLens 2 can be used. It streams or records an egocentric video with an overlay showing the virtual content [27]. Our toolkit supports gaze visualization in this module by attaching a small sphere to the current gaze position that is visible in the capture but not to the user. However, this method is computationally demanding which constrains the framerate for all applications to 30 frames per second and has a negative impact on real-time interactive applications which limits its use to demonstration purposes.

\section{3. $R$ Package for Data Analysis}

We implement an R package for seamless data analysis of recordings from our recording tool. Existing data analysis tools are primarily targeted at stationary eye trackers that yield a two-dimensional gaze signal or mobile eye trackers that report gaze with respect to an egocentric video feed [5,28-30]. Our toolkit reports three dimensional gaze data with a world-centered coordinate system. We provide a new $\mathrm{R}$ package that supports this data paradigm. It offers offline fixation detection with corresponding pre- and post-processing routines. The $\mathrm{R}$ package and detailed documentation is published on GitHub (https:/ / github.com/AR-Eye-Tracking-Toolkit/ARETT-R-Package, accessed on 22 March 2021) under the MIT open-source license.

We implement two functions for pre-processing the raw gaze data, gap fill and noise reduction, similar to Reference [31]. The gap fill function linearly interpolates between valid gaze points with small gaps in between, e.g., due to loss of tracking. The noise reduction function applies a mean or median filter to the gaze data with a given window size.

Three methods from the literature for offline fixation detection are implemented. This includes I-VT using a velocity threshold similar to Reference [31], I-DT for VR as described by Llanes-Jurado et al. [32] using a dispersion threshold, and I-AOI proposed by Salvucci and Goldberg [33] based on detected areas of interest. Our implementation of I-VT follows the description by Olsen [31]. It reproduces a similar behavior based on the data recorded using our toolkit. We calculate a velocity for each gaze point over a specified duration and categorize the points by comparing the velocities to a specified threshold. I-DT follows the implementation by Llanes-Jurado et al. [32]. It computes the angular dispersion distance over a window of a specific size in terms of its duration. If the initial window exceeds this threshold it is moved forward until it does not exceeded the threshold. Then, the window is extended to the right until the dispersion threshold is exceeded. All samples in the window, excluding the last sample, are classified as belonging to a fixation. Afterwards, a new window is initialized at the position of the last gaze sample. These steps are repeated until all samples are classified. The I-AOI method for fixation detection is based on Salvucci and Goldberg [33]. It differs from the other methods as it classifies fixations based on predefined areas of interest. First, all gaze points within an AOI are classified as belonging to a fixation. Next, groups of fixation samples are identified as a fixation event using a minimum duration threshold. Short events are discarded.

In addition, we provide two functions for post-processing of detected fixations: merging adjacent fixations and discarding short fixations. The merge adjacent fixations function 
merges subsequent fixations if the gap is smaller than a defined maximum duration and, depending on the detection algorithm used, a maximum angle between them (I-VT) or a maximum dispersion distance (I-DT). For I-AOI, the two fixations must belong to the same AOI. The discard short fixations function removes short fixations based on a minimum fixation duration and is mainly interesting for the I-VT method because both other methods inherently contain a minimum fixation duration.

\section{Evaluation of Accuracy and Precision}

High eye tracking data quality is important for eye tracking research because errors in the gaze estimation process can undermine the validity of reported results [17]. However, for the integrated eye tracker of the Microsoft HoloLens 2 we only find limited information about spatial accuracy and no information about spatial precision [26]. We conduct a user study to analyze the accuracy and precision of gaze data from the HoloLens 2 that is recorded using our toolkit. We ask participants to fixate a set of targets, which have a static position with respect to the participant's head, at different distances. We record the gaze signal while the participants are seated (setting I) or walking (setting II). Further, we ask them to fixate a target with a static world position while moving around (setting III). The results can serve as a reference for researchers when designing eye tracking studies, e.g., to decide whether the accuracy is sufficient, or to influence the position and size of AOIs. In addition, our results can guide interaction designers that develop gaze-based AR applications, for example to improve gaze-based selection [34].

\subsection{Participants}

In total, we recruited 21 participants (7 or 33\% female; mean age $29.5, S D=8.5$ ) of which 15 participated in all three settings. Two participants skipped setting III and four participants finished setting III only. This totals to 17 participants for settings I and II (4 or $24 \%$ female; mean age $29.1, S D=8.6$ ), and 19 participants for setting III (7 or $37 \%$ female; mean age $30, S D=8.8$ ). All participants had normal or corrected-to-normal vision with one participant wearing contact lenses and three participants wearing glasses.

\subsection{Conditions $\mathcal{E}$ Tasks}

In our study, we include three settings in which we record the participants' gaze signal and the position of multiple fixation targets. In setting I and II, we show a planar 9-point grid of fixation targets $(3 \times 3)$ that is centered in front of the participant's head and orthogonal to the forward direction (Figure 3a). Participants are standing still in setting I, and walking forward and backward in setting II during the recording phase. For both settings, the grid size is aligned to the field of view of the device. The outer fixation targets are positioned at the border of the field of view such that both eyes can see them. The distances between the corner targets (upper left, upper right, lower left, lower right) and the center target are 18.25 degrees of visual angle. The distances for the edge targets (upper center, middle left, middle right, lower center) are 12.13 degrees of visual angle. In addition, we vary the distance $d$ of the grid for both settings: we include $d \in\{0.5 \mathrm{~m}, 1 \mathrm{~m}, 2 \mathrm{~m}, 4 \mathrm{~m}\}$. For all distances, we ask the participants to fixate all targets for three seconds, starting on the upper left in a left-to-right and top-to-bottom direction. An example picture of the settings I and II can be found in Figure 4. For setting III, we place a single fixation target at a static position in the world coordinate system: we show a sphere with diameter of $1 \mathrm{~cm} 15 \mathrm{~cm}$ above the surface on a table with a height of $75 \mathrm{~cm}$ (Figure 3b). Participants are seated in front of the table and are asked to move their heads left and right while keeping up the fixation to the sphere. With this setting, we simulate vestibulo-ocular reflex movements that are common in natural experiment settings in which participants interact with stationary AR content. 


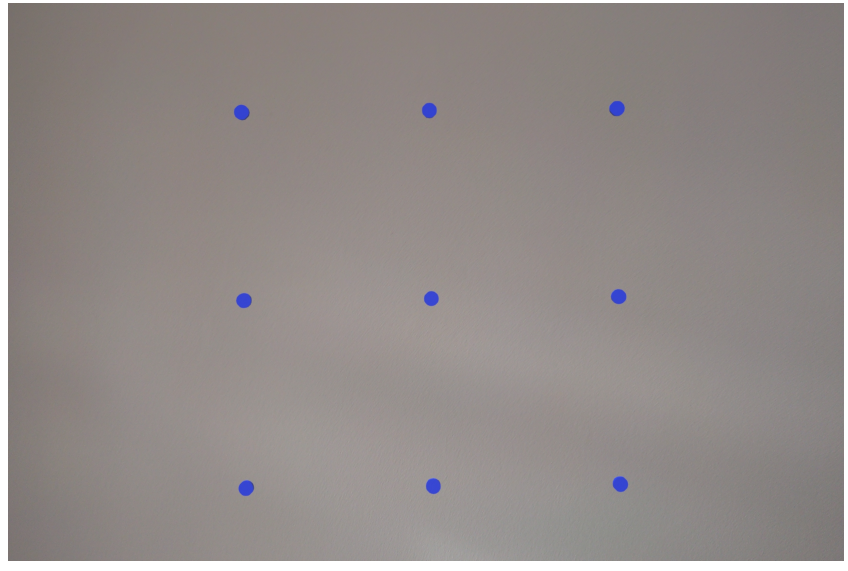

(a) Mixed reality photo of setting I and II

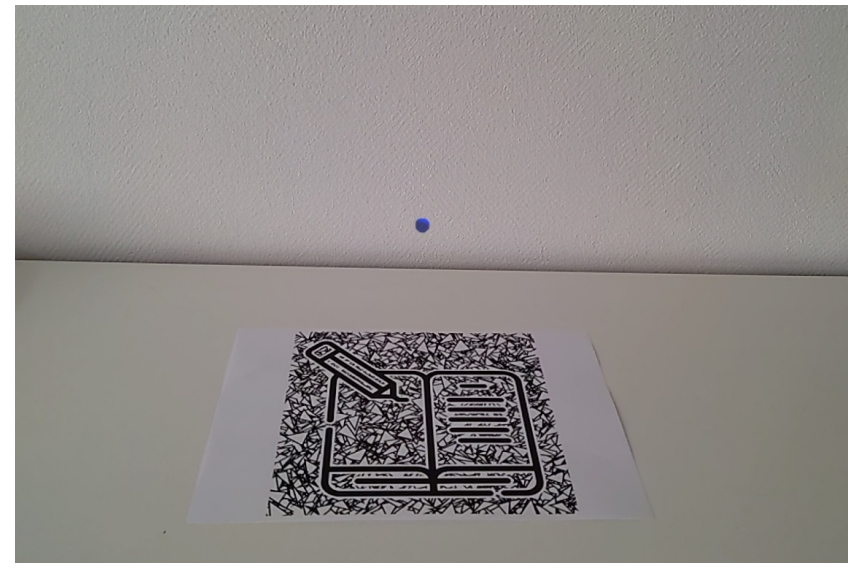

(b) Mixed reality photo of setting III

Figure 3. Mixed reality photo of our HoloLens 2 applications for all three settings which are presented to the participants. The fixation grid for settings I and II is displayed at a fixed distance from the user and resized such that the angular size is identical for all distances (a). The sphere in setting III is positioned $15 \mathrm{~cm}$ above the table and stays fixed on top of the visual marker when the participant moves (b). These screenshots are 2D projections which do not reflect the field-of-view and depth perception of a participant in augmented reality (AR).

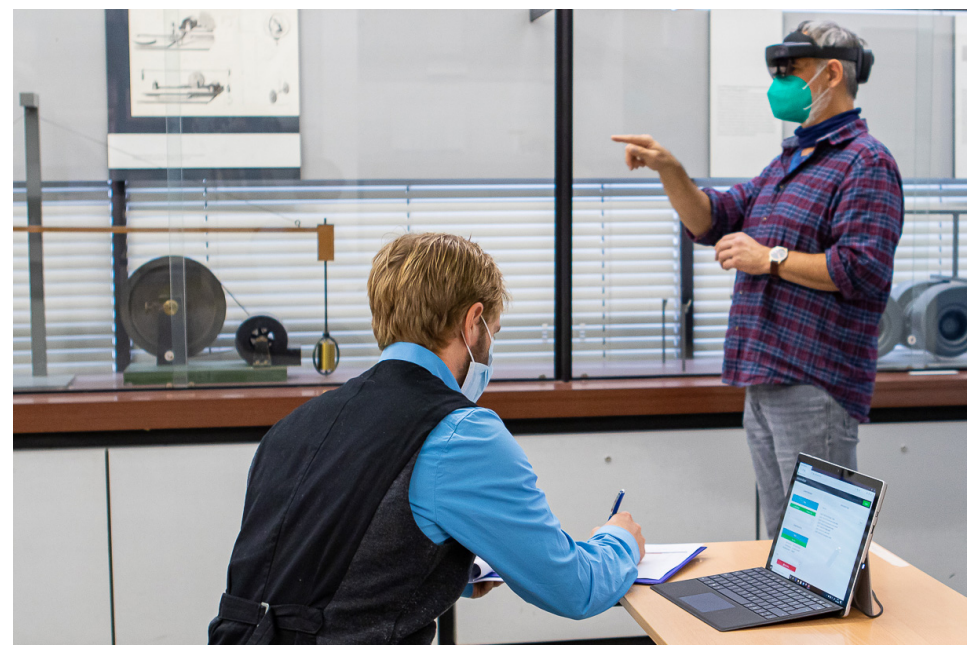

Figure 4. Example of setting I and II in our study with the participant wearing a Microsoft HoloLens 2 and the supervisor controlling the recording using our toolkit.

\subsection{Procedure}

All settings are recorded in one session, starting with setting I and immediately followed by setting II and III. The order of the settings was identical for all participants. In the beginning of a session, the participant puts on the device which is adjusted to the head by a supervisor. The device is fitted to a participant's head such that it does not move during the experiment but is still comfortable to wear. If the participant feels that the device loosens, it is tightened by the supervisor. During the whole procedure, the device is not moved on or removed from the participant's head. After fitting, the integrated eye tracker is calibrated using the built-in calibration routine. We record gaze data and reference target positions with our new toolkit. Each task is recorded separately, resulting in a recording per distance for setting I and II, and a single recording for setting III. For settings I and II, we perform a manual fixation detection and remove gaze samples that belong to a saccade event. We performed a manual annotation of the gaze signal to extract fixations more accurately than possible with automatic algorithms which have, in particular, problems with event detection in mobile eye tracking signals [35]. Gaze samples are labeled as 
belonging to a fixation unless the gaze position moved away from the fixation center, i.e., when turning into a saccade which ends at the next fixation center. The labeling is based on visual inspections from one expert. For setting III, we remove gaze samples before the participant starts fixating the sphere and moving his/her head, and after the participant stops. The participant is asked by the supervisor to start the movement and, after four minutes, asked to stop moving and to return to the starting position.

\subsection{Metrics}

We define spatial accuracy and precision according to the literature $[34,36]$. Per target, we compute spatial accuracy as the distance between the mean gaze sample and the target position. Spatial precision is computed as the standard deviation of the distances between each gaze sample and the mean position of all gaze samples. We report both measures in $\mathrm{cm}$, as well as in degrees of visual angle. The distance in $\mathrm{cm}$ is calculated using the distance between the gaze point and the target based on their positions in the reference frame provided by Unity 3D. The visual angle is calculated as the angle between the reported 3D gaze ray from the gaze origin to the gaze point and the $3 \mathrm{D}$ ray from the gaze origin to the target position.

\subsection{Hypotheses}

Previous research on the gaze estimation error in head-mounted eye tracking reported significant differences in the spatial accuracy for varying distances and when moving around versus resting $[18,20]$. We expect similar characteristics for the integrated eye tracker of the Microsoft HoloLens 2. Hence, we hypothesize that the spatial accuracy is dependent on the distance of the fixation target (H1). Further, we expect a lower accuracy for setting II in which participants move than for setting I in which they are resting $(\mathrm{H} 2)$. Similar to H2, we expect that spatial precision is lower for setting II, i.e., when participants move (H3). For setting III, we exploratively investigate the spatial accuracy and precision for a realistic research setting from educational sciences.

\subsection{Results}

A total of 335,867 gaze points are recorded over all participants in all three settings and before filtering. Analyzing the relative timestamp provided by the device, the mean difference between timestamps is $33 \mathrm{~ms}$ (SD $1 \mathrm{~ms}$ ). One hundred and seventy-one of these gaze points show a time difference to the previous gaze point larger than $34 \mathrm{~ms}$, and 27 gaze points show a difference smaller than $32 \mathrm{~ms}$. Those with a difference larger than $34 \mathrm{~ms}$ are multiples of the expected $33.33 \mathrm{~ms}$. All gaze points with a difference smaller than $32 \mathrm{~ms}$ have a difference of $0 \mathrm{~ms}$. After removing the 198 gaze points with erroneous timing, we see a mean difference between timestamps of $33.33 \mathrm{~ms}$ (SD $2.5 \times 10^{-4} \mathrm{~ms}$ ).

For setting I, we report the metrics for all targets which include, on average, 108.47 $(S D=43.04)$ gaze points after saccade removal. Table 2 shows the spatial accuracy and precision per distance, averaged over all nine fixation targets and participants. The mean angular accuracy over all distances is 0.83 degrees with a precision of 0.27 degrees. Figure 5 visualizes the error for individual targets per distance. A visualization of the analyzed gaze positions of one participant at the upper left target can be found in Figure 6. A Shapiro-Wilk test shows that the means of accuracies in degrees of visual angle over all targets is not distributed normally for all distances but $2.0 \mathrm{~m}, p_{0.5}=0.01, p_{1.0}=0.03$, $p_{2.0}=0.12, p_{4.0}=0.03$. To evaluate the difference in spatial accuracy over all distances we conduct a Friedman test. It shows a significant difference in accuracy between the different distances, $\chi^{2}(3)=20.15, p<0.001$. Post hoc analysis with Wilcoxon signed-rank tests is conducted with a Bonferroni correction applied, resulting in a significance level set at $p<0.008$. It reveals a significant difference in the accuracy between the distance $0.5 \mathrm{~m}$ and the distances $2.0 \mathrm{~m}$ and $3.0 \mathrm{~m}$ (Table 3). 


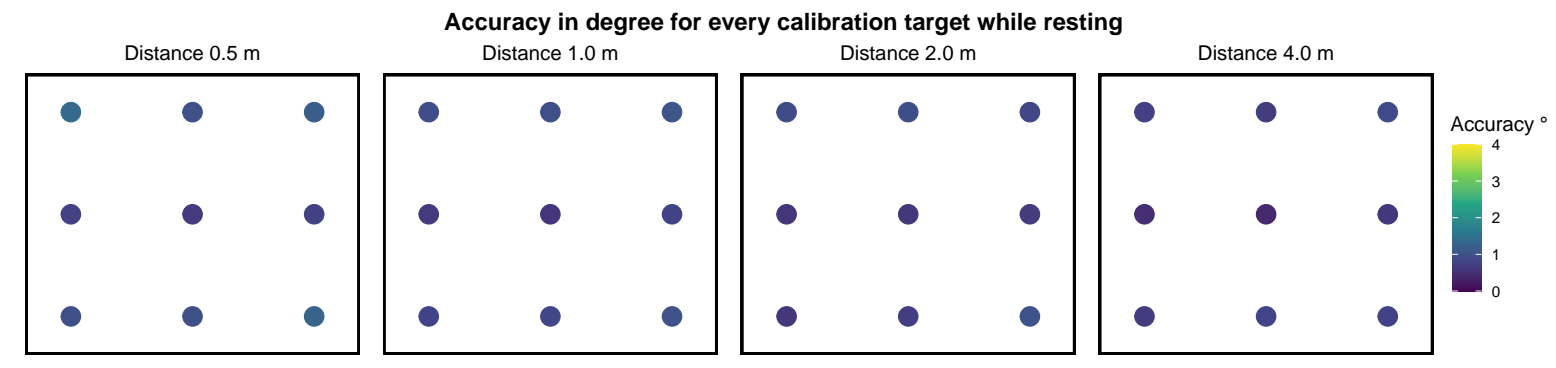

Figure 5. Plot of the mean accuracy at each distance for each target in setting I-resting. The accuracy angle for all targets is smaller than 1.5 degrees.
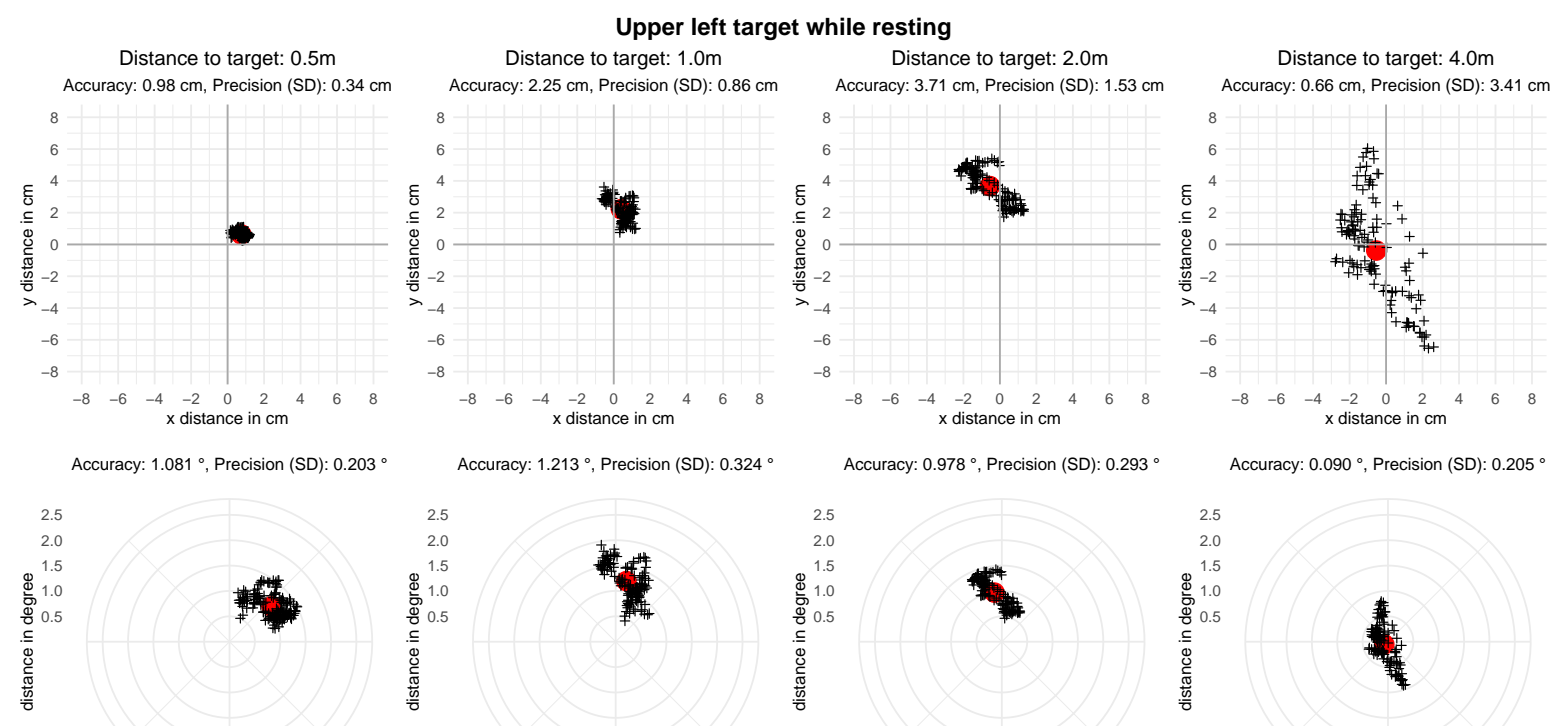

Figure 6. Recorded gaze point of one participant in relation to the upper left target in setting I-resting. The red dot represents the mean gaze position with each cross being one recorded gaze point.

Table 2. Accuracy and precision for setting I-resting.

\begin{tabular}{ccccc}
\hline \multirow{2}{*}{ Distance } & \multicolumn{2}{c}{ Accuracy (SD) } & \multicolumn{2}{c}{ Precision (SD) } \\
& in cm & in deg & in cm & in deg \\
\hline $0.5 \mathrm{~m}$ & $0.91(0.41)$ & $1.00(0.44)$ & $0.40(0.16)$ & $0.29(0.13)$ \\
$1.0 \mathrm{~m}$ & $1.56(0.83)$ & $0.85(0.46)$ & $0.67(0.24)$ & $0.25(0.11)$ \\
$2.0 \mathrm{~m}$ & $2.85(1.31)$ & $0.77(0.35)$ & $1.35(0.49)$ & $0.24(0.10)$ \\
$4.0 \mathrm{~m}$ & $5.03(2.27)$ & $0.68(0.31)$ & $3.12(1.26)$ & $0.28(0.12)$ \\
\hline
\end{tabular}

Table 3. Results of the post hoc Wilcoxon signed-rank tests for setting I-resting. * the Bonferroni corrected significane level is $p<0.008$.

\begin{tabular}{|c|c|c|c|c|c|c|}
\hline Comparison & $\begin{array}{c}0.5 \mathrm{~m} \\
-1.0 \mathrm{~m}\end{array}$ & $\begin{array}{c}0.5 \mathrm{~m} \\
-2.0 \mathrm{~m}\end{array}$ & $\begin{array}{c}0.5 \mathrm{~m} \\
-4.0 \mathrm{~m}\end{array}$ & $\begin{array}{c}1.0 \mathrm{~m} \\
-2.0 \mathrm{~m}\end{array}$ & $\begin{array}{c}1.0 \mathrm{~m} \\
-4.0 \mathrm{~m}\end{array}$ & $\begin{array}{c}2.0 \mathrm{~m} \\
-4.0 \mathrm{~m}\end{array}$ \\
\hline $\mathrm{Z}$ & -2.63 & -3.57 & -3.43 & -1.68 & -2.06 & -1.44 \\
\hline $\mathrm{p}$ & 0.009 & $<0.001$ * & 0.001 * & 0.093 & 0.039 & 0.149 \\
\hline
\end{tabular}

The recordings for setting II include an average of $121.23(S D=32.53)$ gaze samples per target. The mean spatial accuracy, averaged over participants and fixation targets per distance, is reported in Table 4 . The mean angular accuracy over all distances is 1.77 degrees with a precision of 1.13 degrees. The results per fixation target are visualized in Figure 7. 
A visualization of the analyzed gaze positions of one participant at the upper left target can be found in Figure 8 . The mean accuracy in degrees of visual angle over all targets is distributed normally for the distances $0.5 \mathrm{~m}$ and $4.0 \mathrm{~m}$, but not at $1.0 \mathrm{~m}$ and $2.0 \mathrm{~m}$ as assessed by a Shapiro-Wilk test, $p_{0.5}=0.44, p_{1.0}=0.01, p_{2.0}=0.04, p_{4.0}=0.35$. Analogue to setting I we conduct a Friedman test to evaluate the difference in spatial accuracy over all distances. It shows a significant difference in accuracy between the different distances, $\chi^{2}(3)=37.02, p<0.001$. The Bonferroni corrected post hoc analysis with Wilcoxon signed-rank tests results in a significance level set at $p<0.008$. It reveals a significant difference in spatial accuracy for all paired comparisons except for the distances $2.0 \mathrm{~m}$ and $4.0 \mathrm{~m}$ (Table 5).

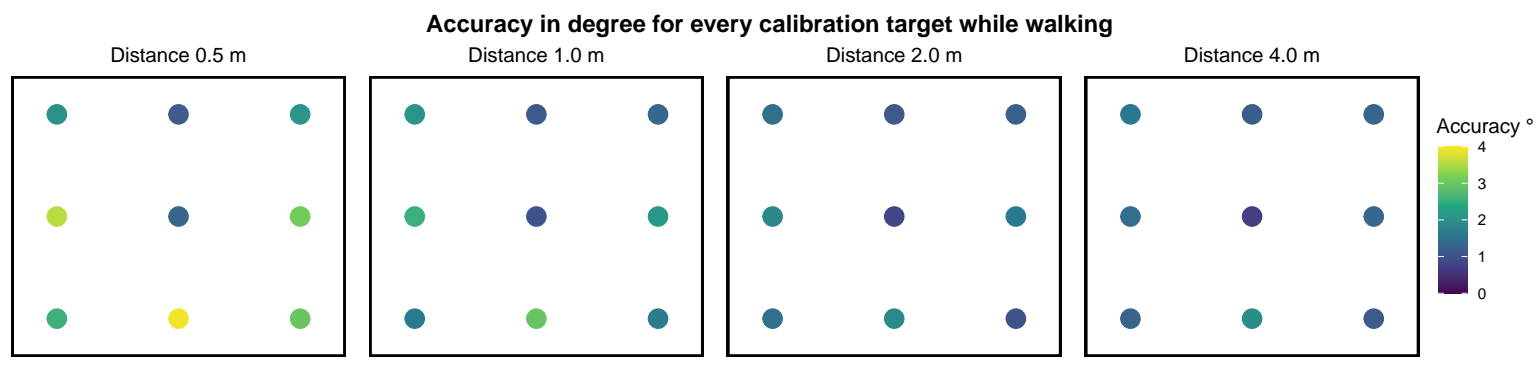

Figure 7. Plot of the mean accuracy at each distance for each target in setting II-walking.
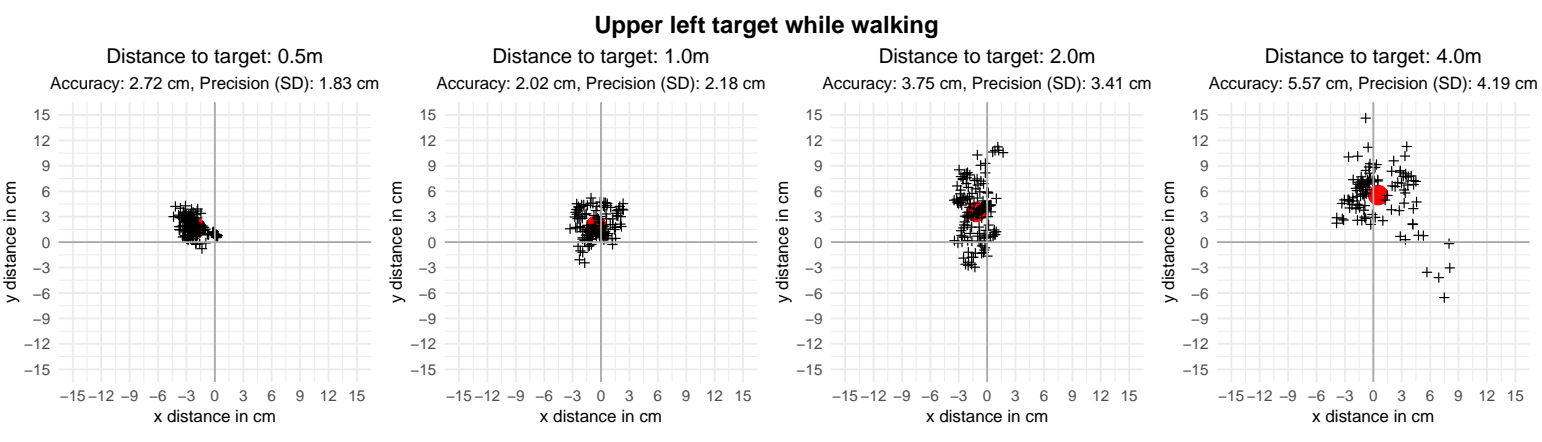

Accuracy: $2.672^{\circ}$, Precision (SD): $1.206^{\circ}$

Accuracy: $1.045^{\circ}$, Precision (SD): $0.680^{\circ}$

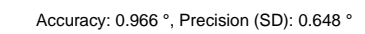

Accuracy: $0.742^{\circ}$, Precision (SD): $0.284^{\circ}$
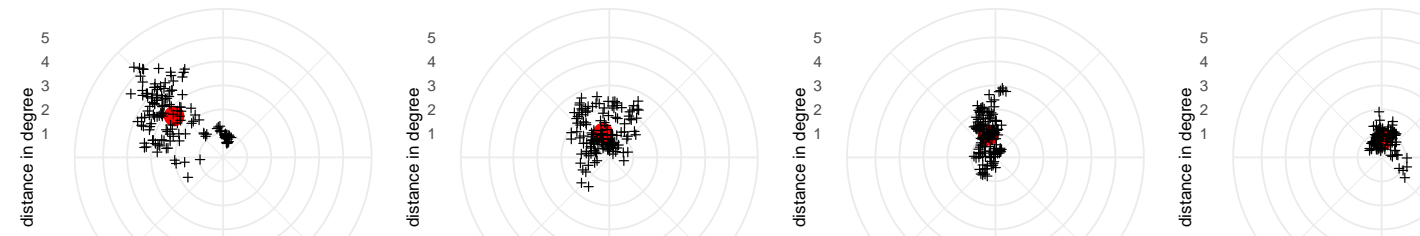

Figure 8. Recorded gaze point of one participant in relation to the upper left target in setting II-walking. The red dot represents the mean gaze position with each cross being one recorded gaze point.

Table 4. Accuracy and precision for setting II-walking.

\begin{tabular}{ccccc}
\hline \multirow{2}{*}{ Distance } & \multicolumn{2}{c}{ Accuracy (SD) } & \multicolumn{2}{c}{ Precision (SD) } \\
& in cm & in deg & in cm & in deg \\
\hline $0.5 \mathrm{~m}$ & $2.29(0.64)$ & $2.52(0.69)$ & $1.89(0.34)$ & $1.31(0.25)$ \\
$1.0 \mathrm{~m}$ & $3.35(1.50)$ & $1.84(0.81)$ & $3.33(1.00)$ & $1.16(0.47)$ \\
$2.0 \mathrm{~m}$ & $5.07(1.94)$ & $1.39(0.53)$ & $6.32(1.52)$ & $1.03(0.27)$ \\
$4.0 \mathrm{~m}$ & $9.75(3.08)$ & $1.33(0.42)$ & $12.58(3.19)$ & $1.03(0.32)$ \\
\hline
\end{tabular}


Table 5. Results of the post hoc Wilcoxon signed-rank tests for setting II-walking. * the Bonferroni corrected significance level is $p<0.008$.

\begin{tabular}{|c|c|c|c|c|c|c|}
\hline Comparison & $\begin{array}{c}0.5 \mathrm{~m} \\
-1.0 \mathrm{~m}\end{array}$ & $\begin{array}{c}0.5 \mathrm{~m} \\
-2.0 \mathrm{~m}\end{array}$ & $\begin{array}{c}0.5 \mathrm{~m} \\
-4.0 \mathrm{~m}\end{array}$ & $\begin{array}{c}1.0 \mathrm{~m} \\
-2.0 \mathrm{~m}\end{array}$ & $\begin{array}{c}1.0 \mathrm{~m} \\
-4.0 \mathrm{~m}\end{array}$ & $\begin{array}{c}2.0 \mathrm{~m} \\
-4.0 \mathrm{~m}\end{array}$ \\
\hline Z & -3.432 & -3.621 & -3.621 & -3.574 & -2.817 & -0.686 \\
\hline $\mathrm{p}$ & $0.001 *$ & $<0.001^{*}$ & $<0.001^{*}$ & $<0.001 *$ & $0.005 *$ & 0.492 \\
\hline
\end{tabular}

In addition, we compare the spatial accuracy and precision results between setting I (resting) and setting II (walking). The differences in accuracy are not distributed normally for the distances $0.5 \mathrm{~m}$ and $1.0 \mathrm{~m}$ as assessed by a Shapiro-Wilk test, $p_{0.5}=0.04, p_{1.0}=0.003$, $p_{2.0}=0.26, p_{4.0}=0.44$. A Wilcoxon signed-rank test shows that the accuracy differs significantly between setting I and II for all distances (Table 6). The difference in precision is distributed normally for the distance of $0.5 \mathrm{~m}$ but not for the other distances as assessed by a Shapiro-Wilk test, $p_{0.5}=0.44, p_{1.0}<0.001, p_{2.0}=0.046, p_{4.0}=0.003$. A Wilcoxon signed-rank test shows that the precision differs significantly between setting I and II for all distances (Table 7).

Table 6. Results of the Wilcoxon signed-rank tests for the comparison of the accuracy between setting I and II.

\begin{tabular}{ccccc}
\hline Distance & $\mathbf{0 . 5} \mathbf{~ m}$ & $\mathbf{1 . 0} \mathbf{~ m}$ & $\mathbf{2 . 0} \mathbf{~ m}$ & $\mathbf{4 . 0 ~} \mathbf{~}$ \\
\hline $\mathrm{Z}$ & -3.62 & -3.62 & -3.57 & -3.53 \\
$\mathrm{p}$ & $<0.001$ & $<0.001$ & $<0.001$ & $<0.001$ \\
\hline
\end{tabular}

Table 7. Results of the Wilcoxon signed-rank tests for the comparison of the precision between setting I and II.

\begin{tabular}{ccccc}
\hline Distance & $\mathbf{0 . 5} \mathbf{~ m}$ & $\mathbf{1 . 0} \mathbf{~ m}$ & $\mathbf{2 . 0} \mathbf{~ m}$ & $\mathbf{4 . 0 ~} \mathbf{~}$ \\
\hline $\mathrm{Z}$ & -3.62 & -3.62 & -3.62 & -3.62 \\
$\mathrm{p}$ & $<0.001$ & $<0.001$ & $<0.001$ & $<0.001$ \\
\hline
\end{tabular}

For setting III, we include a mean of $641.79(S D=262.10)$ gaze samples per participant for our analysis. The resulting accuracy and precision values together with the mean distance of the participants from the target can be found in Table 8 . We approximate the spatial accuracy in degrees of visual angle as using the following formula: $\theta=\tan ^{-1}(O / d)$ with the accuracy in $\mathrm{cm}$ as $O$ and the mean distance to the participant $d$. The same formula is used to calculate the precision by using the precision in $\mathrm{cm}$ as $O$. A 3D visualization of the analyzed gaze positions of one participant can be found in Figure 9.

Table 8. Accuracy, precision, and mean distance for setting III-stationary target.

\begin{tabular}{ccccc}
\hline $\begin{array}{c}\text { Distance (SD) } \\
\text { in cm }\end{array}$ & in cm & in deg & in cm & in deg \\
\hline $49.87(13.53)$ & $0.34(0.27)$ & $0.39(0.31)$ & $0.87(0.35)$ & $1.00(0.40)$ \\
\hline
\end{tabular}


Stationary target

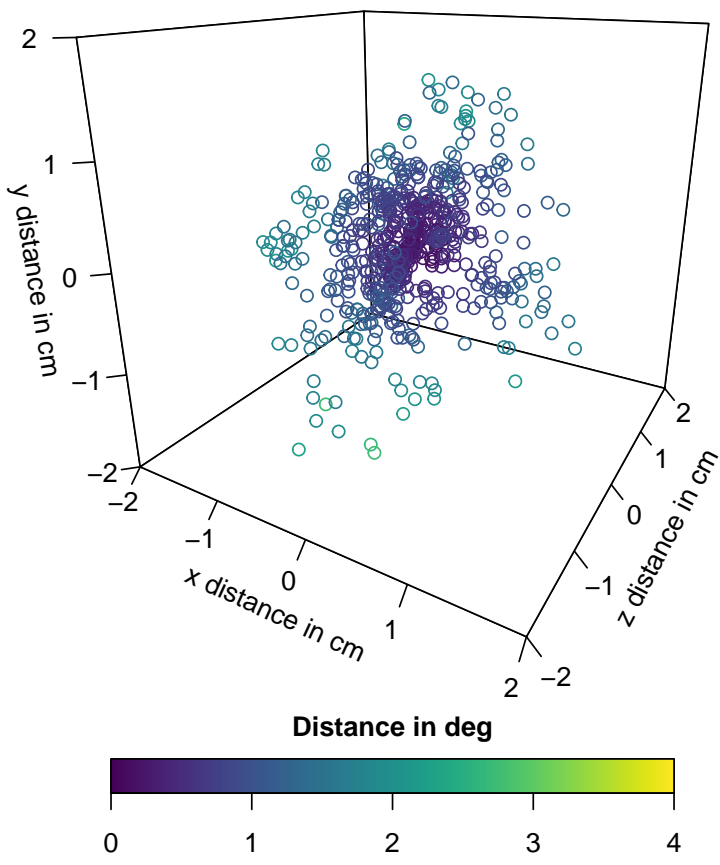

Figure 9. Recorded gaze point of one participant in setting III—stationary target. The distance angle for all gaze points is smaller than 3 degrees.

\section{Discussion}

The major goal of developing the augmented reality eye tracking toolkit is to enable researchers to easily use eye tracking in AR settings with the HoloLens 2. It should allow an efficient integration to Unity 3D scenes, enable recordings of a comprehensive set of eye tracking signals (see Table 1), and a seamless analysis of the data via our R package. This would simplify integration of eye tracking into existing AR research, like Strzys et al. [37] and Kapp et al. [38]. Independently from the study reported in this publication, our toolkit is currently being used in two ongoing research studies which provide first evidences in this direction. One study utilizes the Microsoft HoloLens 2 to display two dimensional plots at a fixed distance while the participant is moving while another study investigates stationary augmentations on a table. The initial feedback from the study organizers, the developers of the AR application, and the experimenters is positive. No major issues occurred during the recordings, which certifies a high robustness, and the ease-of-use of the web interface was, informally, rated high.

Our toolkit can also be used for facilitating gaze-based interaction and real-time adaptive applications using the data provider module. For instance, prior research proposed to use eye tracking and HMDs to augment the episodic memory of dementia patients by storing artificial memory sequences and presenting them when needed [39]. Other works include approaches for gaze-based analysis of the users' attention engagement and cognitive states for proactive content visualization [40], and multi-focal plane interaction, such as object selection and manipulation at multiple fixation distances [41]. It can also be used in research regarding selection techniques in AR [42,43]. The utility of the toolkit for realizing real-time adaptive applications has been shown in Reference [44]. The presented prototype uses video and gaze information via our toolkit to automatically recognize and augment attended objects in an uninstrumented environment. 


\subsection{Evaluation of Accuracy and Precision}

The results from our evaluation show significant differences in spatial accuracy for varying distances in setting I and II. This supports our hypothesis H1. However, for setting $\mathrm{I}$, the pairwise comparisons reveal that only the results for the smallest distance $0.5 \mathrm{~m}$ and the distances 2.0 and $4.0 \mathrm{~m}$ differ significantly. For setting II, the results significantly differ for all pairs except for the two farthest distances of $2.0 \mathrm{~m}$ and $4.0 \mathrm{~m}$. Further, our results confirm the hypothesis $\mathrm{H} 2$ and $\mathrm{H} 3$ : the accuracies and precision for each distance differ significantly between setting I and setting II while the results for setting II are poorer.

Our observations also show that the spatial accuracy in degrees of visual angle increases with increasing distance (see Tables 2 and 4). Findings from the literature suggest that the accuracy decreases with increasing deviation from the calibration distance, i.e., the distance at which the fixation targets of the calibration routine are shown $[18,20,45]$. This leads to our assumption that the built-in calibration routine of HoloLens 2 is placed at 2 to $4 \mathrm{~m}$ from the user, which is supported by the fact that Microsoft recommends an interaction distance of $2 \mathrm{~m}$ [46]. It is possible that this increase in angular accuracy is an effect of the vergence-accommodation conflict [47] as only a combined gaze ray is made available by the device.

The official HoloLens 2 documentation reports a vague range for the spatial accuracy of "approximately within 1.5 degrees" with "slight imperfections" to be expected [26]. Basically, our results coincide with these specifications, but are much more fine-grained. For the resting setting (I), we observe better spatial accuracy values ranging from 1.00 degrees of visual angle for a $0.5 \mathrm{~m}$ distance to 0.68 degrees for $4.0 \mathrm{~m}$. For the walking setting (II), which has a lower spatial accuracy overall, the results for $0.5 \mathrm{~m}$ and $1.0 \mathrm{~m}$ are outside the official range with 2.52 and 1.84 degrees of visual angle, respectively. The two other conditions lie within the specified boundary of 1.5 degrees. The documented sampling

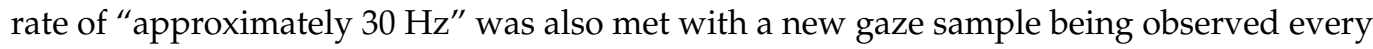
$33.33 \mathrm{~ms}$.

Based on our findings, we suggest minimum target sizes for eye tracking research and gaze-based interaction with the HoloLens 2. Similar to Feit et al. [34], who investigate the gaze estimation error for remote eye tracking, we calculate the minimum size such that $95 \%$ of all gaze samples hit the target. We use their formula that computes the minimum size based on a 2-dimensional Gaussian function as $S=2(O+2 \sigma)$ with the spatial accuracy of the eye tracker as offset $O$ and the spatial precision of the gaze signal as $\sigma$. The resulting minimum target sizes for varying distances are listed in Table 9 . For a distance of $2.0 \mathrm{~m}$, Microsoft recommends a target size of 5-10 cm, which conforms with our findings for setting I: we suggest a target size of $11.10 \mathrm{~cm}$ in this case. However, if the participant is meant to move around, the targets should be significantly larger.

Table 9. Recommended minimum target size in $\mathrm{cm}$ based on Feit et al. [34] and the identified accuracy and precision.

\begin{tabular}{ccc}
\hline Distance & Setting I (Resting) & Setting II (Walking) \\
\hline $0.5 \mathrm{~m}$ & $3.42 \mathrm{~cm}$ & $12.14 \mathrm{~cm}$ \\
$1.0 \mathrm{~m}$ & $5.80 \mathrm{~cm}$ & $20.02 \mathrm{~cm}$ \\
$2.0 \mathrm{~m}$ & $11.10 \mathrm{~cm}$ & $35.42 \mathrm{~cm}$ \\
$4.0 \mathrm{~m}$ & $22.54 \mathrm{~cm}$ & $69.82 \mathrm{~cm}$ \\
\hline
\end{tabular}

In setting III, we explore the characteristics of the gaze estimation error for stationary targets. The average distance to the stationary target of $49.87 \mathrm{~cm}$ is comparable to the $0.5 \mathrm{~m}$ distance in setting I. However, the mean spatial accuracy is better and the precision is lower. This better result for spatial accuracy could be explained by the longer fixation durations and the varying viewing angles in setting III: on average, the mean gaze positions seem to balance around the fixation target, while the dispersion stays high (see Figure 9). Based on Feit et al. [34], we suggest a minimum target size of $4.16 \mathrm{~cm}$. This is $22 \%$ larger than the recommendation for setting I, and $34 \%$ of the recommended size for setting II. Altogether, 
the results suggest that the fixation duration and the user condition, i.e., walking versus not walking, influences the spatial accuracy and precision, which should be considered when designing interactive and, potentially, mobile research applications.

Finally, we compare the results of the HoloLens 2 eye tracker to available headmounted eye trackers without an HMD. Macinnes et al. [48] evaluated the spatial accuracy and precision of three mobile eye trackers for multiple distances while participants were seated. They included (i) the Pupil Labs $120 \mathrm{~Hz}$ Binocular glasses with an accuracy of $0.84^{\circ}$ and a precision of $0.16^{\circ}$, (ii) the SensoMotoric Instruments (SMI) Eye Tracking Glasses 2 with an accuracy of $1.21^{\circ}$ and a precision of $0.19^{\circ}$, and (iii) the Tobii Pro Glasses 2 with an accuracy of $1.42^{\circ}$ and a precision of $0.34^{\circ}$. On average, our results for the HoloLens 2 in setting I, which is the closest match to the setting in Reference [48], yield an accuracy of $0.83^{\circ}$ and a precision of $0.27^{\circ}$. This is similar to the results of the Pupil Labs glasses that ranged best in the experiment by Macinnes et al. [48] and suggests that the eye tracking data from HoloLens 2 can effectively be used in research experiments. However, one drawback is that the sampling rate of $30 \mathrm{~Hz}$ is lower compared to the devices evaluated in their experiment.

\subsection{Limitations}

Our toolkit enables access to raw gaze data and provides additional tools for processing them. However, it is limited to the data that is made available through APIs of the device. For instance, the API reports a joint gaze vector for both eyes, while many commercial binocular eye tracking glasses report separate gaze rays. This forces to intersect the gaze ray with the virtual environment to receive a point of gaze. Separate rays can be intersected to extract gaze points without intersecting any surface, and to infer the fixation depth. In addition, this gaze point can be used to find close-by AOIs. Our evaluation focuses on limited set of interaction settings that probably do not generalize to all possible settings in AR environments. However, with setting III, we include a more realistic setting that closer matches typical AR environments with a moving user and fixed visualizations. We cannot rule out effects due to the experiment order as it was identical for all participants.

Currently, our toolkit is constrained to the Microsoft HoloLens 2 as eye tracking device. However, all device specific functionality is encapsulated in the data access layer. This makes it possible to adapt the toolkit to other eye tracking enabled AR devices in the future. However, the gaze estimation is device-specific: the results from our evaluation on spatial accuracy and spatial precision do not hold for other devices. In addition, the sampling rate might change which needs to be addressed by re-configuring the data pulling rate. The data access layer could also subscribe gaze events or connect to a signal stream, if this is supported by the new device.

\section{Conclusions}

In this work, we presented an open-source toolkit that enables eye tracking research in AR using the HoloLens 2 device. We addressed the gap of missing research tools by implementing a Unity 3D package for reliable gaze data acquisition and an $\mathrm{R}$ package for seamless data analysis. We received first positive feedback on our toolkit from two other research studies, proving its utility. We conducted a user study $(n=21)$ to investigate the spatial accuracy and spatial precision of gaze data from our toolkit. The results suggest that the spatial accuracy increases when increasing the distance of fixation targets. Further, we found evidence that spatial accuracy and precision drop when participants are walking compared to standing still. Overall, the gaze estimation error is similar to recent headmounted eye trackers without HMDs which shows the suitability of our toolkit for research applications. In future updates we will address the limitations of our toolkit as follows. We plan to add fully integrated support for video recording of the integrated camera using the data logger, as well as real-time streaming of video and gaze data. We will also investigate the effectiveness of attaching virtual AOIs to real objects for real-time gaze-to-AOI mapping. 
Further, we want to extend the functionality of our R package and integrate interfaces to existing gaze data processing tools, as well as integrate data access layers for other devices.

Author Contributions: Conceptualization, methodology, software, formal analysis, data curation, visualization, supervision, S.K.; investigation, S.K. and S.M.; writing-original draft preparation, S.K. and M.B.; writing-review and editing, S.K., M.B., S.M., D.S., J.K.; resources, J.K.; project administration, funding acquisition, D.S. and J.K. All authors have read and agreed to the published version of the manuscript.

Funding: This research was funded by the German Federal Ministry of Education and Research (Bundesministerium für Bildung und Forschung; BMBF) via the project "GeAR" (Grant No. 01JD1811B and 01JD1811C).

Institutional Review Board Statement: Not applicable.

Informed Consent Statement: Informed consent was obtained from all subjects involved in the study.

Data Availability Statement: The data presented in this study are available on request from the corresponding author. The data are not publicly available due to data privacy.

Conflicts of Interest: The authors declare no conflict of interest. The funders had no role in the design of the study; in the collection, analyses, or interpretation of data; in the writing of the manuscript, or in the decision to publish the results.

\section{References}

1. Majaranta, P.; Bulling, A. Eye Tracking and Eye-Based Human-Computer Interaction. In Advances in Physiological Computing; Fairclough, S.H., Gilleade, K., Eds.; Human-Computer Interaction Series; Springer: London, UK, 2014; pp. 39-65. [CrossRef]

2. Blattgerste, J.; Renner, P.; Pfeiffer, T. Advantages of eye-gaze over head-gaze-based selection in virtual and augmented reality under varying field of views. In Proceedings of the Workshop on Communication by Gaze Interaction-COGAIN '18; Morimoto, C., Pfeiffer, T., Eds.; ACM Press: New York, NY, USA, 2018; pp. 1-9. [CrossRef]

3. Guenter, B.; Finch, M.; Drucker, S.; Tan, D.; Snyder, J. Foveated 3D graphics. ACM Trans. Graph. 2012, 31, 1-10. [CrossRef]

4. Patney, A.; Salvi, M.; Kim, J.; Kaplanyan, A.; Wyman, C.; Benty, N.; Luebke, D.; Lefohn, A. Towards foveated rendering for gaze-tracked virtual reality. ACM Trans. Graph. 2016, 35, 1-12. [CrossRef]

5. Tobii Pro AB. Pro Lab User Manual. Available online: https://www.tobiipro.com/siteassets/tobii-pro/user-manuals/Tobii-ProLab-User-Manual/?v=1.152 (accessed on 12 November 2020).

6. Pupil Labs. Add Awareness to Your VR/AR Experience: Integrate and React. Available online: https://pupil-labs.com/ products/vr-ar/ (accessed on 20 November 2020).

7. Tobii VR. Tobii VR: Discover New Possibilities with Eye Tracking in VR. Available online: https://vr.tobii.com/ (accessed on 20 November 2020).

8. Stratmann, T.C.; Gruenefeld, U.; Boll, S. EyeMR_Low-cost Eye-Tracking for Rapid-prototyping in Head-mounted Mixed Reality. In Proceedings of the 2018 ACM Symposium on Eye Tracking Research \& Applications; Sharif, B., Krejtz, K., Eds.; ACM: New York, NY, USA, 2018; pp. 1-2. [CrossRef]

9. Lee, K.F.; Chen, Y.L.; Yu, C.W.; Chin, K.Y.; Wu, C.H. Gaze Tracking and Point Estimation Using Low-Cost Head-Mounted Devices. Sensors 2020, 20, 1917. [CrossRef] [PubMed]

10. Mardanbegi, D.; Pfeiffer, T. EyeMRTK: A Toolkit for Developing Eye Gaze Interactive Applications in Virtual and Augmented Reality. In Proceedings of the 11th ACM Symposium on Eye Tracking Research E Applications; Krejtz, K., Sharif, B., Eds.; ACM: New York, NY, USA, 2019; pp. 1-5. [CrossRef]

11. Adhanom, I.B.; Lee, S.C.; Folmer, E.; MacNeilage, P. GazeMetrics: An Open-Source Tool for Measuring the Data Quality of HMD-based Eye Trackers. In ACM Symposium on Eye Tracking Research and Applications; Bulling, A., Huckauf, A., Jain, E., Radach, R., Weiskopf, D., Eds.; ACM: New York, NY, USA, 2020; pp. 1-5. [CrossRef]

12. Magic Leap. Magic Leap 1: A Thousand Breakthroughs in One. Available online: https://www.magicleap.com/en-us/magicleap-1 (accessed on 20 November 2020).

13. Microsoft. HoloLens 2: A New Reality for Computing. Available online: https://www.microsoft.com/en-us/hololens (accessed on 20 November 2020).

14. Microsoft. Eye Tracking in the Mixed Reality Toolkit. Available online: https://microsoft.github.io/MixedRealityToolkit-Unity/ Documentation/EyeTracking/EyeTracking_Main.html (accessed on 17 November 2020).

15. Magic Leap. Eye Gaze. Available online: https://developer.magicleap.com/en-us/learn/guides/design-eye-gaze (accessed on 20 November 2020).

16. Hausamann, P.; Sinnott, C.; MacNeilage, P.R. Positional head-eye tracking outside the lab: An open-source solution. In ACM Symposium on Eye Tracking Research and Applications; Bulling, A., Huckauf, A., Jain, E., Radach, R., Weiskopf, D., Eds.; ACM: New York, NY, USA, 2020; pp. 1-5. [CrossRef] 
17. Holmqvist, K.; Andersson, R. Eye Tracking: A Comprehensive Guide to Methods, Paradigms and Measures; Lund Eye-Tracking Research Institute: Lund, Sweden, 2011.

18. Mardanbegi, D.; Hansen, D.W. Parallax error in the monocular head-mounted eye trackers. In Proceedings of the 2012 ACM Conference on Ubiquitous Computing; ACM: New York, NY, USA, 2012; pp. 689-694. [CrossRef]

19. Barz, M.; Stauden, S.; Sonntag, D. Visual Search Target Inference in Natural Interaction Settings with Machine Learning. In Proceedings of the 2020 ACM Symposium on Eye Tracking Research E Applications; Association for Computing Machinery: New York, NY, USA, 2020; pp. 1-8. [CrossRef]

20. Barz, M.; Daiber, F.; Bulling, A. Prediction of Gaze Estimation Error for Error-Aware Gaze-Based Interfaces. In Proceedings of the Ninth Biennial ACM Symposium on Eye Tracking Research \& Applications; ACM Press: New York, NY, USA, 2016; pp. 275-278. [CrossRef]

21. Holmqvist, K.; Nyström, M.; Mulvey, F. Eye tracker data quality: What it is and how to measure it. In Proceedings of the Symposium on Eye Tracking Research and Applications; ACM: New York, NY, USA, 2012; pp. 45-52. [CrossRef]

22. Barz, M.; Bulling, A.; Daiber, F. Computational Modelling and Prediction of Gaze Estimation Error for Head-Mounted Eye Trackers; Technical Report; DFKI: Kaiserslautern, Germany, 2015.

23. Unity Technologies. Unity Real-Time Development Platform I3D, 2D VR \& AR Engine. Available online: https://unity.com/ (accessed on 23 February 2021).

24. The R Foundation. R: The R Project for Statistical Computing. Available online: https://www.r-project.org/ (accessed on 23 February 2021).

25. Microsoft. EyesPose Class. Available online: https://docs.microsoft.com/de-de/uwp/api/windows.perception.people. eyespose?view=winrt-19041 (accessed on 17 November 2020).

26. Microsoft. Eye Tracking on HoloLens 2. Available online: https://docs.microsoft.com/en-us/windows/mixed-reality/design/ eye-tracking (accessed on 12 November 2020).

27. Microsoft. Create Mixed Reality Photos and Videos. Available online: https://docs.microsoft.com/en-us/hololens/holographicphotos-and-videos (accessed on 13 November 2020).

28. Kassner, M.; Patera, W.; Bulling, A. Pupil: An Open Source Platform for Pervasive Eye Tracking and Mobile Gaze-based Interaction. In Proceedings of the 2014 ACM International Joint Conference on Pervasive and Ubiquitous Computing Adjunct PublicationUbiComp '14 Adjunct; Brush, A.J., Friday, A., Kientz, J., Scott, J., Song, J., Eds.; ACM Press: New York, NY, USA, $2014 ;$ pp. 1151-1160. [CrossRef]

29. Dink, J.; Ferguson, B. eyetrackingR: An R Library for Eye-tracking Data Analysis. Available online: http:/ / www.eyetracking-r. com/ (accessed on 24 November 2020).

30. Zhegallo, A.V.; Marmalyuk, P.A. ETRAN-R Extension Package for Eye Tracking Results Analysis. Perception 2015, 44, 1129-1135. [CrossRef] [PubMed]

31. Olsen, A. The Tobii I-VT Fixation Filter: Algorithm description. Available online: https://www.tobiipro.com/siteassets/tobiipro/learn-and-support/analyze/how-do-we-classify-eye-movements/tobii-pro-i-vt-fixation-filter.pdf/?v=2012 (accessed on 12 November 2020).

32. Llanes-Jurado, J.; Marín-Morales, J.; Guixeres, J.; Alcañiz, M. Development and Calibration of an Eye-Tracking Fixation Identification Algorithm for Immersive Virtual Reality. Sensors 2020, 20, 4956. [CrossRef] [PubMed]

33. Salvucci, D.D.; Goldberg, J.H. Identifying Fixations and Saccades in Eye-Tracking Protocols. In Proceedings of the Eye Tracking Research E Applications Symposium 2000 Palm Beach Gardens, FL, November 6-8, 2000; Association for Computing Machinery: New York, NY, USA, 2000. [CrossRef]

34. Feit, A.M.; Williams, S.; Toledo, A.; Paradiso, A.; Kulkarni, H.; Kane, S.; Morris, M.R. Toward Everyday Gaze Input. In Proceedings of the 2017 CHI Conference on Human Factors in Computing Systems; Mark, G., Fussell, S., Lampe, C., Schraefel, M., Hourcade, J.P., Appert, C., Wigdor, D., Eds.; ACM: New York, NY, USA, 2017; pp. 1118-1130. [CrossRef]

35. Steil, J.; Huang, M.X.; Bulling, A. Fixation detection for head-mounted eye tracking based on visual similarity of gaze targets. In Eye Tracking Research and Applications Symposium (ETRA); Association for Computing Machinery: New York, NY, USA, 2018; pp. 1-9. [CrossRef]

36. Duchowski, A.; Medlin, E.; Cournia, N.; Murphy, H.; Gramopadhye, A.; Nair, S.; Vorah, J.; Melloy, B. 3-D eye movement analysis. Behav. Res. Methods Instrum. Comput. 2002, 34, 573-591. [CrossRef] [PubMed]

37. Strzys, M.P.; Kapp, S.; Thees, M.; Kuhn, J.; Lukowicz, P.; Knierim, P.; Schmidt, A. Augmenting the thermal flux experiment: A mixed reality approach with the HoloLens. Phys. Teach. 2017, 55, 376-377. [CrossRef]

38. Kapp, S.; Thees, M.; Strzys, M.P.; Beil, F.; Kuhn, J.; Amiraslanov, O.; Javaheri, H.; Lukowicz, P.; Lauer, F.; Rheinländer, C.; et al. Augmenting Kirchhoff's laws: Using augmented reality and smartglasses to enhance conceptual electrical experiments for high school students. Phys. Teach. 2019, 57, 52-53. [CrossRef]

39. Orlosky, J.; Toyama, T.; Sonntag, D.; Kiyokawa, K. Using Eye-Gaze and Visualization to Augment Memory. In Distributed, Ambient, and Pervasive Interactions; Streitz, N., Markopoulos, P., Eds.; Springer International Publishing: Cham, Switzerland, 2014; Volume 8530 LNCS, pp. 282-291. [CrossRef]

40. Toyama, T.; Sonntag, D.; Orlosky, J.; Kiyokawa, K. Attention Engagement and Cognitive State Analysis for Augmented Reality Text Display Functions. In Proceedings of the 20th International Conference on Intelligent User Interfaces-IUI '15; ACM Press: New York, NY, USA, 2015; pp. 322-332. [CrossRef] 
41. Toyama, T.; Orlosky, J.; Sonntag, D.; Kiyokawa, K. A Natural Interface for Multi-Focal Plane Head Mounted Displays Using 3D Gaze. In Proceedings of the 2014 International Working Conference on Advanced Visual Interfaces; Association for Computing Machinery: New York, NY, USA, 2014; pp. 25-32. [CrossRef]

42. van der Meulen, H.; Kun, A.L.; Shaer, O. What Are We Missing? In ISS '17: Proceedings of the 2017 ACM International Conference on Interactive Surfaces and Spaces; Association for Computing Machinery: New York, NY, USA, 2017; pp. 396-400. [CrossRef]

43. Kytö, M.; Ens, B.; Piumsomboon, T.; Lee, G.A.; Billinghurst, M. Pinpointing. In Proceedings of the 2018 CHI Conference on Human Factors in Computing Systems—CHI '18; Mandryk, R., Hancock, M., Perry, M., Cox, A., Eds.; ACM Press: New York, NY, USA, 2018; pp. 1-14. [CrossRef]

44. Barz, M.; Kapp, S.; Kuhn, J.; Sonntag, D. Automatic Recognition and Augmentation of Attended Objects in Real-time using Eye Tracking and a Head-mounted Display. Manuscript submitted for publication.

45. Cerrolaza, J.J.; Villanueva, A.; Villanueva, M.; Cabeza, R. Error characterization and compensation in eye tracking systems. In Proceedings of the Symposium on Eye Tracking Research and Applications; ACM: New York, NY, USA, 2012; pp. 205-208. [CrossRef]

46. Microsoft. Comfort. Available online: https://docs.microsoft.com/de-de/windows/mixed-reality/design/comfort (accessed on 25 November 2020).

47. Kramida, G. Resolving the Vergence-Accommodation Conflict in Head-Mounted Displays. IEEE Trans. Vis. Comput. Graph. 2016, 22, 1912-1931. [CrossRef] [PubMed]

48. Macinnes, J.J.; Iqbal, S.; Pearson, J.; Johnson, E.N. Wearable Eye-tracking for Research: Automated dynamic gaze mapping and accuracy/precision comparisons across devices. bioRxiv 2018. [CrossRef] 